\title{
Découverte de services basée sur leurs protocoles de conversation
}

\author{
Juan Carlos Corrales — Daniela Grigori - Mokrane Bouzeghoub \\ Laboratoire PRiSM, Université de Versailles Saint-Quentin en Yvelines \\ 45 avenue des Etats-Unis, 78035 Versailles Cedex, FRANCE \\ \{Juan-Carlos.Corrales-Munoz,Daniela.Grigori,Mokrane.Bouzeghoub\}@prism.uvsq.fr
}

\begin{abstract}
RÉSUMÉ. La découverte de services utiles à une application devient de plus en plus critique dans plusieurs domaines. Les approches de découverte actuelles, basées sur l'appariement des entrées/sorties, ou même complétées par des ontologies, sont limitées car elles ne prennent pas en compte l'aspect sémantique de ces services. Aussi, la sélectivité de ces approches reste très faible; il revient à l'utilisateur de naviguer sur les nombreux résultats pour retrouver les services qui l'intéressent. Dans cet article, nous affirmons qu'une approche basée sur le comportement des services(en particulier leurs protocoles de conversation) peut aider à sélectionner les meilleurs services et à réduire l'effort de navigation de l'utilisateur. Ceci est d'autant plus important que la découverte peut se faire de façon programmée et dynamique. L'approche proposée se base sur un appariement sémantique des graphes de processus, délivrant aussi bien des résultats exacts que des résultats approchés. Des opérations d'édition permettent de modifier le graphe requête pour le rapprocher le plus possible des graphes cibles selon une distance associée à chaque requête. Nous illustrons notre approche par un exemple basé sur des protocoles de conversation exprimés en utilisant le langage WSCL.

ABSTRACT. The capability to easily find useful services becomes increasingly critical in several fields. Current approaches for services retrieval are mostly limited to the matching of their inputs/outputs, possibly annotated with ontologies. Recent works have demonstrated that this approach is not sufficient to discover relevant components. In this paper we argue that, in many situations, the service discovery should be based on the specification of service behavior (in particular, the conversation protocol). The idea behind is to develop matching techniques that operate on behavior models and allow delivery of partial matches as well as their semantic distance with user requirements. A set of edit operations alter query graph to make it as close as possible to target graphs, depending on a distance fixed for each query graph. We exemplify our approach for conversation protocol matchmaking by using the WSCL language.
\end{abstract}

MOTS-CLÉS : services web, découverte de services, appariement de graphes

KEYWORDS: web services, services retrieval, graph matching.

ISI. Volume $8-\mathrm{n}^{\circ} 2 / 2005$, pages 1 à 15 


\section{Introduction}

La découverte de services utiles à une application (composants logiciels, services Web, calculs scientifiques) devient de plus en plus critique dans plusieurs domaines où les seuls noms de services ou leurs entrées/sorties ne sont pas suffisants à leur identification. Des exemples de tels services sont nombreux :

- Progiciels spécialisés, publiés sur Internet comme des services web et qui peuvent être invoqués à distance via des interfaces utilisateurs ou des interfaces programmes (API). Un des principaux problèmes des modèles de services web est de mettre en correspondance les demandeurs de services et les fournisseurs de services ; en particulier dans un environnement fortement dynamique, comme le Web, où les services sont fréquemment modifiés, publiés ou retirés.

- Programmes de calcul scientifique qui sont des ressources très importantes dans une architecture de type grille de calcul, quelquefois encore plus importantes que les données elles-mêmes (Foster et al., 2003; Foster et al., 2002). Dans ces systèmes, données et procédures sont des objets de première importance; ils peuvent être publiés, recherchés et composés avec d'autres. Les scientifiques, qui sont les principaux utilisateurs de ces architectures, procèdent par exploration et évaluation des procédures qui les intéressent afin de retenir celles satisfaisant certaines caractéristiques et éviter ainsi des développements longs et coûteux.

- Composants logiciels disponibles dans des bibliothèques de composants génériques ou métier, conçus pour être réutilisés dans un processus d'ingénierie d'applications. L'ingénierie par réutilisation réduit les coûts de développement, de test et de maintenance ou d'évolution.

Dans tous ces cas, les utilisateurs, finaux ou développeurs, sont intéressés par la découverte de composants dans des catalogues ou des bibliothèques comportant aussi bien le code des composants que leur spécification sous forme de modèles. L'utilisateur formule son besoin comme un modèle de processus qui va servir de requête destinée au système de découverte de composants qui lui fournira tous les composants identiques ou partiellement similaires à sa requête. Sur la base de ces résultats, il pourra sélectionner le ou les composants les plus adaptés (en sémantique et en qualité) à ses besoins. Même si le choix se porte sur des composants similaires, la modification ou l'adaptation de ces derniers peut s'avérer moins couteuse que leur développement intégral.

Dans cet article, nous affirmons qu'une approche basée sur la sémantique des processus (en particulier leurs protocoles de conversation) peut aider à sélectionner les meilleurs services et à réduire l'effort de navigation de l'utilisateur. Ceci est d'autant plus important que la découverte peut se faire de façon programmée et dynamique n'impliquant pas nécessairement l'utilisateur. L'approche que nous développons est basée sur un appariement sémantique des graphes de processus, délivrant, en cas d'absence de processus équivalents, tous ceux dont la spécification satisfait une certaine distance sémantique fixée pour la requête, que nous appellerons des appariements partiels. Notre problème se pose donc en termes d'appariement de graphes où des opé- 
rations d'édition permettent de modifier le graphe requête pour le rapprocher le plus possible des graphes cibles selon une distance associée à chaque requête. Nous proposons un ensemble d'opérations primitives permettant d'adapter l'algorithme d'appariement à une grande variété de graphes de processus. Nous illustrons notre approche par un exemple basé sur des protocoles de conversation extraits du standard WSCL.

La section suivante présente quelques scénarios applicatifs motivant l'intérêt de notre approche. La section 3 présente les approches existantes de découverte de services et montre leurs limites par rapprot à nos exemples de scénarios. Dans la section 4, nous montrons comment la découverte de service basée sur le comportement des processus peut être ramenée à un appariement de graphes. Une mesure de similarité, basée sur des opérations d'édition de graphes, est introduite. La section 5 décrit notre algorithme d'appariement de graphe, dérivé de (Messmer, 1995), ainsi que les adaptations qui en ont été faites pour tenir compte des protocoles de conversation. La section 6 présente une implémentation de cet algorithme et une étude expérimentale de ses performances. Enfin, la section 7 présente nos conclusions et nos travaux futurs.

\section{Motivations par quelques scénarios applicatifs}

Dans cette section, nous présentons deux scénarios nécessitant une découverte de services, donc un appariement de graphes. Le premier exemple se situe dans le contexte des services web et consiste à rechercher les services ayant des comportements compatibles pour les intégrer. Le second scénario concerne la détection de différences entre deux modèles de processus, appelée aussi analyse différentiellle.

Intégration de services web. Considérons une compagnie qui utilise un service $\mathrm{S}$ pour commander des fournitures de bureau. Elle cherche à trouver les détaillants ayant des services web compatibles pour permettre leur composition. Les interactions offertes par un service web sont spécifiées au moment de la conception, en utilisant ce qui s'appelle un protocole de conversation (Benatallah et al., 2004b). Les protocoles de conversation indiquent les séquences d'échanges de message qui sont permises par le service, exprimés en termes de contraintes sur l'ordre dans lequel les opérations de service devraient être invoquées. Ils peuvent être exprimés à l'aide de standards comme BPEL ou WSCL ou tout autre langage de protocoles (voir par exemple (Benatallah et al., 2004b)). La spécification des protocoles de conversation est importante car il est rare que les opérations réalisées par les services puissent être invoquées dans n'importe quel ordre et indépendamment les unes des autres. La recherche de services basée sur la compatibilité de leurs protocoles de conversation est donc un point important dans l'intégration de services. Parmi les services offerts par les détaillants, seuls ceux ayant des protocoles compatibles doivent être proposés. Si de tels services n'existent pas, il faut diriger la recherche vers des services ayant des protocoles proches mais pour lesquels, il sera nécessaire de développer un ou plusieurs adaptateurs permettant l'interaction avec ces services. La découverte de protocoles compatibles permet une réutilisation sans effort des services correspondants alors que 
la découverte de protocoles proches peut déclencher la génération semi-automatique d'adaptateurs (voir (Benatallah et al., 2005)).

Comme exemple, considérons un premier protocole de conversation défini par la séquence suivante : les clients se connectent (login) pour demander un catalogue des produits (getCatalog), suivi par une commande d'article incluant les options et préférences de livraison, (submitOrder, sendShippingPreferences), et enfin par une demande de facturation (issueInvoice) et un ordre de paiement (makePayment) (voir Figure 1). Le second protocole est décrit par la séquence d'opérations suivante : login, getCatalog, submitOrder, issueInvoice, makePayment et sendShippingPreferences où les préférences de livraison sont données à la fin du processus. Ceci est possible si la facturation ne dépend pas des préférences de livraison, auquel cas ces dernières peuvent être spécifiées n'importe où dans le processus.
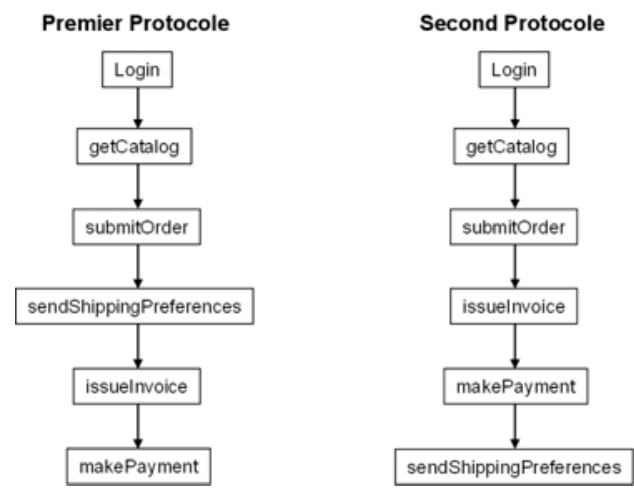

Figure 1. Deux protocoles de conversation

Le processus de recherche doit comparer les deux processus et montrer le degré de similarité entre leurs graphes correspondants, malgré l'ordre différent de leurs opérations. Dans le cas de cet exemple, un adaptateur rétablissant l'ordre des opérations doit être généré en utilisant, par exemple, les techniques présentées dans (Benatallah et al., 2005). Il faut noter que si l'appariement est limité aux entrées/sorties, on ne pourrait pas mettre en évidence la différence de protocoles de conversation et la nécessité de définir un adaptateur n' aurait pas émergé.

Analyse différentielle. L'analyse différentielle consiste à trouver les différences entre deux modèles. Par exemple, comparer un modèle implanté dans une entreprise par rapport à un modèle métier standardisé. C'est la cas, par exemple, des standards PIPs définis par RosettaNet et utilisé par les entreprises affiliées. Dans ce contexte, une validation systématique doit être faite entre les processus d'entreprise et les modèles standards en confrontant les deux types de modèles pour isoler ainsi les éléments de l'application qui ne sont pas conformes aux prescriptions des standards. Basée sur ces différences, une évaluation doit être faite pour estimer si un processus de ré-ingénierie doit être initié pour aligner le processus d'entreprise aux standards. 


\section{Etat de l'art}

Actuellement, les algorithmes de découverte de services dans les registres tels que UDDI ou ebXML sont basés sur une recherche par mots clés ou des tables de correspondances regroupant des couples (clé, valeur). Dans le cadre du Web sémantique, des logiques de description ont été proposées pour une définition formelle plus riche et plus précise des services. Ces langages tels que, par exemple, OWL-S, permettent la définition d'ontologies qui servent de base à l'appariement sémantique de services (Paolucci et al., 2002; Bernstein et al., 2002; Benatallah et al., 2003). Dans (Paolucci et al., 2002; Benatallah et al., 2003), l'appariement entre un service demandé et un service offert est basé sur les entrées/sorties des deux services, regardant en particulier s'ils ont les mêmes types ou des types hiérarchiquement inclus les uns dans les autres.

Dans (Kawamura et al., 2003), des filtres séparés ont été définis pour la recherche de services, basée sur : l'espace des noms, la description textuelle, l'ontologie du domaine utilisée, les types des entrées/sorties, les contraintes, etc. L'approche présentée dans (Cardoso et al., 2003) prend en compte le temps d'exécution des services, leur coût d'utilisation et leur fiabilité. Les auteurs de (Wu et al., 2005) fournissent un algorithme léger de comparaison sémantique des services, basé sur une méthode d'estimation de la similarité à différents niveaux (lexical, qualité de service, interface).

Dans le contexte des grilles de calcul (Foster et al., 2002), la recherche de services est basée sur un langage de haut niveau permettant l'expression de relations entre les services, d'une part, et leurs entrées/sorties, d'autre part.

La recherche de service basée sur des mots-clés ou quelques attributs sémantiques n'est pas satisfaisante pour un grand nombre d'applications. La tendance des travaux récents est d'exploiter des connaissances sur la sémantique et le comportement des services. Le besoin de prendre en compte le comportement décrit par un modèle de processus a été souligné par plusieurs travaux (Trastour et al., 2001; S. Bansal et al., 2003; Bernstein et al., 2002; Zdravkovic et al., 2004; Piccinelli et al., 2001; Wombacher et al., 2004).

Dans (Bernstein et al., 2002), l'amélioration de la découverte de services est faite à travers les propriétés les plus importantes du comportement des processus. Les modèles de processus ont une expressivité plus grande que les mots clés, offrant ainsi une plus grande précision dans la comparaison des services. Un langage de requêtes pour découvrir des services a été défini; il permet de spécifier des requêtes à l'aide de conditions sur les activités qui composent les services, les exceptions traitées et les flots de données entre les activités.

Dans (S. Bansal et al., 2003), les auteurs motivent les faiblesses des appariements basés sur les entrées/sorties en montrant que certains résultats dépendent de conditions internes qui, si elles ne sont pas explicitées, peuvent conduire à des erreurs grossières d'appariement. Ils proposent un algorithme qui tient compte de la structure des processus, en particulier des conditions de branchement. 
Dans (Trastour et al., 2001), les auteurs montrent bien l'importance de l'aspect comportemental dans l'appariement de processus métiers B2B mais ne situent leurs solutions que dans des perspectives de recherche. Il en est de même des auteurs de (Piccinelli et al., 2001) qui proposent un modèle d'agrégation dynamique de services ; ils pointent clairement la nécessité d'inclure comme une exigence la spécification de la dynamique des processus pour garantir leur compatibilité dans le contexte des marchés électroniques.

Dans (Zdravkovic et al., 2004), les auteurs traitent de l'équivalence de deux processus spécifiés par des réseaux de Petri. On suppose que les partenaires se découvrent mutuellement via un registre de services métier, puis vérifient la concordance de leurs protocoles par rapport à ceux existant dans l'entreprise.

Dans (Wombacher et al., 2004), les auteurs donnent une sémantique formelle à leur algorithme d'appariement de services, basée sur l'intersection des automates d'états finis étendus par des expressions logiques associées aux états. Le calcul des intersections des automates est très coûteux et ne peut passer à l'échelle lorsqu'on a des catalogues de services volumineux. Pour résoudre ce problème, les auteurs de (Wombacher et al., 2005) proposent une approche d'indexation pour interroger des processus métiers itératifs (ayant des boucles) stockés dans une base de données. Ils introduisent une fonction d'abstraction qui élimine les cycles et transforme une séquence potentiellement infinie de messages en une représentation finie pouvant être gérée par un SGBD. Les limites de cette approche consistent dans le choix d'une représentation sous forme d'automate d'états fini. Cette représentation limite l'expressivité du modèle, en particulier pour la représentation du parallélisme qui peut conduire à des modèles ayant un grands nombre d'états.

Très récemment, différents articles ont été publiés sur la similarité et la compatibilité à différents niveaux d'abstraction de la description des services (Benatallah et al., 2004a; Bordeaux et al., 2004; Dong et al., 2004; Ponnekanti et al., 2004; Wombacher et al., 2004). Ces approches proposent des modèles basés sur le pi-calcul ou des machines d'états et des mécanismes de comparaison de spécifications des services pour vérifier leur compatibilité.

Un nouveau modèle de comportement pour les services web est présenté dans (Shen et al., 2005). Les services web sont modélisés comme des automates finis non déterministes. La description du comportement du service web inclut à la fois les messages echangés par le service et les activités réalisée dans le service. Les activités sont decrites en utilisant OWL-S (Web Services Ontology Language). Les auteurs proposent un nouveau langage de requêtes permettant d'exprimer des propriétés temporelles et semantiques des services et des algorithmes pour l'évaluation des requêtes.

Pour résumer, le besoin de tenir compte du comportement lors de la découverte de services a été souligné par de nombreux auteurs et quelques propositions récentes présentent un intérêt certain (Shen et al., 2005),(Wombacher et al., 2004). Mais elles restent guidées par une approche exacte de la recherche et ne permettent pas de pro- 
poser des services proches qui ne s'apparient pas exactement avec la requête mais qui peuvent être aisément adaptés au besoin de l'utilisateur.

Notre objectif est de proposer une approche de découverte de services basée sur leur comportement et permettant un appariement approximatif, capable de sélectionner aussi bien les services répondant exactement au besoin que ceux répondant partiellement. A notre connaissance, à part les premières idées que nous avons exposées dans (Grigori et al., n.d.), une telle approche n'a pas encore été proposée. Les sections suivantes décrivent les fondements et l'algorithme de cette approche ainsi que la mesure de similarité que nous avons retenue pour sélectionner les services proches de la requête.

\section{Une approche basée sur les graphes pour l'appariement des services}

Dans cette section nous montrons comment l'appariement basé sur le comportemental est réduit à un problème d'appariement de graphes. La section 4.1 rappelle les principes de la méthode d'appariement de graphes que nous utilisons, l'isomorphisme de sous-graphe avec correction d'erreur, qui est basé sur l'idée d'opérations d'édition de graphe. Les prochaines sections montrent comment nous l'adaptons à notre problème : nous élargissons l'ensemble d'opérations d'édition de graphes et nous montrons comment composer deux graphes pour répondre à des exigences de l'utilisateur.

Un protocole de conversation décrit le comportement observable d'un service Web. Il complète la définition de l'interface du service Web en imposant des contraintes sur l'ordre des messages échangés. La plupart des propositions existantes (des standards et des modèles de recherches) sont basés sur des graphes. Pour cette raison, nous choisissons d'utiliser une représentation à base de graphes de protocoles de conversation afin de comparer deux modèles. La représentation des services sous forme de graphes permet de ramener le problème d'appariement de services à un problème d'appariement de graphes. Nous voulons comparer le graphe du processus représentant les exigences de l'utilisateur (appelé graphe requête) avec les graphes offerts dans le catalogue de services (appelés graphes cibles). Le processus d'appariement peut alors être reformulé comme une recherche d'isomorphisme de graphes ou de sous-graphes. Cependant, l'isomorphisme exact de graphes ou de sous-graphes n'est pas satisfaisant dans la mesure où le graphe requête ne correspond pas à une exigence exacte de l'utilisateur mais à une intention qu'il faut interpréter de façon non restrictive. Ainsi, il faut aussi proposer des modèles cibles qui ont une structure semblable lorsqu'il n'existe pas de structures identiques. L'appariement de graphes avec correction d'erreur intègre le concept de recherche approximative dans le processus d'appariement ((Shapiro et al., 1981; Bunke, 2000)). Dans la section suivante nous rappelons brièvement le principe de cette méthode d'appariement de graphes et les définitions de base telles que données dans (Messmer, 1995). 


\subsection{Fondements et définitions de base}

Afin de comparer les graphes cibles à un graphe requête et décider de leurs similarités, il est nécessaire de définir une mesure de distance pour les graphes. De manière similaire au problème d'appariement de chaînes de caractères où des opérations d'édition sont utilisées pour définir la distance d'édition entre deux chaines, la distance d'edition de sous-graphe est basée sur l'idée d'opérations d'édition qui sont appliquées au graphe requête. Les opérations d'édition sont utilisées pour changer le graphe requête jusqu'à ce qu'il existe un isomorphisme de sous-graphes au graphe cible. Pour chaque opération d'édition, un certain coût est assigné. Les coûts sont dépendants de l'application et reflètent la probabilité de restructuration des graphes. Plus une certaine restructuration est probable de se produire, plus son coût est petit. La distance d'édition de sous-graphes d'un graphe requête à un graphe cible est alors définie comme le coût minimum de toutes les séquences d'opérations d'édition qui sont nécessaires pour obtenir un isomorphisme de sous-graphes. On peut conclure que plus la distance de sous-graphes entre un graphe requête et un graphe cible est petite, plus les graphes sont similaires. Les opérations d'édition de graphe considérées sont les suivantes : modifications des étiquettes des sommets et des arcs, insertion et suppression des sommets, insértion et suppression des arrêtes.

Dans la suite nous donnons les définitions pour l'appariement de graphe avec correction d'erreur reprises de (Messmer, 1995).

Un graphe étiqueté orienté est défini par un quadruplet $G=(V, E, \alpha, \beta)$ :

- $V$ est l'ensemble de sommets

- $E \subset V \times V$ est l'ensemble d'arcs

- $\alpha: V \rightarrow L_{V}$ est une fonction affectant des étiquettes aux sommets

- $\beta: E \rightarrow L_{E}$ est une fonction affectant des étiquettes aux arcs.

Définition 1 (Isomorphisme de graphes). Soit $g$ et $g^{\prime}$ deux graphes. Un isomorphisme de graphes entre $g$ et $g^{\prime}$ est une application bijective $f: V \rightarrow V^{\prime}$ tel que

- $\alpha(v)=\alpha^{\prime}(f(v))$ pour tout $v \in V$

- pour chaque arc $e=(u, v) \in E$ il existe un arc $e^{\prime}=(f(u), f(v)) \in E^{\prime}$ tel que $\beta(e)=\beta^{\prime}\left(e^{\prime}\right)$ et pour chaque arc $e^{\prime}=\left(u^{\prime}, v^{\prime}\right) \in E^{\prime}$ il existe un arc $e=$ $\left(f^{-1}(u), f^{-1}(v)\right) \in E$ tel que $\beta(e)=\beta^{\prime}\left(e^{\prime}\right)$.

Si $f: V \rightarrow V^{\prime}$ est un isomorphisme de graphes entre $g$ et $g^{\prime}$, et $g^{\prime}$ est le sousgraphe d'un graphe $g^{\prime \prime}$, i.e. $g^{\prime} \subset g^{\prime}$ ", alors $f$ est appelé un isomorphisme de sousgraphes de $g$ à $g$ ".

Etant donné un graphe $G$, une opération d'édition $\delta$ sur $G$ est une des opérations suivantes :

- remplacement de l'étiquette $\alpha(v)$ du sommet $v$ par $l$

- remplacement de l'étiquette $\beta(e)$ de l'arc $e$ par $l^{\prime}$ 
- suppression du sommet $v$ de $G$ (pour la correction des sommets manquants). Remarquons que tous les arcs incidents du sommet $v$ sont supprimés aussi.

- suppression de l'arc $e$ de $G$ (pour la correction des arcs manquants).

- insertion d'un arc entre deux sommets existants (pour la correction des arcs supplémentaires, c'est-à-dire, les arcs entre deux sommets du deuxième graphe qui ne sont pas présents dans le premier graphe).

Définition 2 (Graphe édité). Etant donné un graphe et une opération d'édition $\delta$, le graphe édité $\delta(G)$ est le graphe dans lequel l'opération $\delta$ a été appliquée (modification de l'étiquette d'un sommet ou d'un arc, suppression d'un arc ou d'un sommet, insertion d'un arc). Etant donné un graphe $G$ est une séquence d'opérations d'édition $\Delta=\left(\delta_{1}, \delta_{2}, \cdots \delta_{k},\right)$, le graphe édité $\Delta(G)$ est le graphe $\Delta(G)=\delta_{k}\left(\cdots \delta_{2}\left(\delta_{1}(G)\right)\right)$..).

Définition 3 (Isomorphisme de sous-graphes avec tolérance d'erreur). Etant donnés deux graphes $G$ et $G^{\prime}$, un isomorphisme de sous-graphes avec tolérance d'erreur (te) $f$ de $G$ à $G^{\prime}$ est un doublet $f=\left(\Delta, f_{\Delta}\right)$ où $f_{\Delta}$ est une séquence d'opérations d'édition et $f$ est un isomorphisme de sous-graphes de $\Delta(G)$ à $G^{\prime}$.

A chaque opération d'édition $\delta$ est assigné un coût $C(\delta)$. Le coût d'un isomorphisme de sous-graphes avec tolérance d'erreur $f=\left(\Delta, f_{\Delta}\right)$ est le coût des opérations d'édition $\Delta$, i.e., $C(\Delta)=\sum_{i=1}^{k} C\left(\delta_{i}\right)$. En général, il existe plusieurs séquences d'opérations d'édition conduisant à plusieurs isomorphismes de sous-graphes. Nous nous intéressons à l'isomorphisme de sous-graphes avec un coût minimal.

Définition 4 (Distance d'édition de sous-graphe). Soit $G$ et $G^{\prime}$ deux graphes. La distance d'édition de sous-graphes de $G$ à $G^{\prime}, \operatorname{de}\left(G, G^{\prime}\right)$ est donnée par le coût minimum de tous les isomorphismes de sous-graphes $f$ de $G$ à $G^{\prime}$.

\subsection{Extension de la distance d'édition de sous-graphes}

Dans (Messmer, 1995), les opérations d'édition de graphes suivantes sont considérées : modification des étiquettes des sommets et des arcs, suppression et insertion des arcs, suppression des sommets.

Les modèles à comparer peuvent avoir différents niveaux de granularité pour réaliser la même fonctionnalité. Par exemple, le premier service a une opération simple (activité) pour réaliser une certaine fonctionnalité, alors que dans le deuxième service le même comportement est réalisé en composant plusieurs opérations. Ainsi, de nouvelles opérations d'édition de graphes sont nécessaires. Etant donné un graphe $G$, nous élargissons la définition des opérations d'édition $\delta \operatorname{sur} G$ en ajoutant deux opérations :

- décomposition d'un sommet $v$ en deux sommets $v_{1}, v_{2}$

- jointure de deux sommets $v_{1}, v_{2}$ dans un nouveau sommet $v$

Nous nous limitons à un cas simple de décomposition, où un sommet est décomposé en une séquence de deux sommets. Ce type simple de décomposition est suffisant 
pour les applications que nous avons analysées. Une opération plus générale serait de décomposer un sommet en un sous-graphe connexe plus complexe; ceci fait l'objet de travaux futurs.

L'opération de décomposition d'un sommet $v$ en deux sommets $v 1, v 2$ est exécutée de la manière suivante :

- tous les arcs ayant comme destination le sommet $v$ auront comme destination le sommet $v_{1}$;

- tous les arcs ayant comme source le sommet $v$ auront comme source le sommet $v_{2}$

- un arc sera ajouté entre les sommets $v_{1}$ et $v_{2}$.

L'opération de jointure est exécutée d'une manière similaire. Ces deux nouvelles opérations d'édition permettent de modéliser des mappings de type 1-N entre les sommets des deux graphes (c.-à-d., un sommet dans le premier graphe correspond à deux sommets dans l'autre graphe). Les opérations classiques d'édition ne prennent en compte que des dépendances de type 1-1 entre les sommets des deux graphes. Par exemple, si un sommet $v$ dans le premier graphe correspond à la composition de deux sommets dans le deuxième graphe ( $v 1$ suivi par $v 2$ ), un algorithme d'appariement basé sur la distance d'édition classique trouverait un mapping entre $v$ et $v 1$ et supprimerait $v 2$. Il ne serait pas possible de découvrir que $v$ correspond à une composition de $v 1$ et $v 2$.

\subsection{Composition de plusieurs graphes cibles pour l'appariement du graphe d'entrée}

Si pour deux graphes cibles, les isomorphismes trouvés correspondent a des sousgraphes disjoints du graphe requête, alors il est possible de les composer pour obtenir un graphe qui couvre mieux les besoins de l'utilisateur.

Considérons deux graphes cible $G_{1}$ et $G_{2}$. Soit $f_{1}=\left(\Delta_{1}, f_{\Delta 1}\right)$ et $f_{2}=\left(\Delta_{2}, f_{\Delta 2}\right)$ deux isomorphismes de sous-graphes de $G_{1}$ et $G_{2}$ à $G_{I}$, respectivement. Le problème est de trouver un isomorphisme de sous-graphes de $G=G_{1} U G_{2}$ à $G_{I}$ qui se base sur $f_{1}$ et $f_{2}$. $f_{1}$ et $f_{2}$ peuvent être combinées s'il n'y a pas deux noeuds dans $\Delta\left(G_{1}\right)$ et $\Delta\left(G_{2}\right)$ qui sont appariés au même noeud du graphe d'entrée. Plus précisément, l'intersection des images de $f_{\Delta 1}$ et $f_{\Delta 2}$ doit être vide, c'est-à-dire, $f_{\Delta 1}\left(V_{1}\right) \cap f_{\Delta 2}\left(V_{2}\right)=\phi$. Pour construire un isomorphisme de sous-graphes avec tolérance d'erreurs $f=\left(\Delta, f_{\Delta}\right)$ à partir de $f_{1}$ et $f_{2}$, il faut générer un ensemble d'opérations d'édition $\Delta$ et un isomorphisme de sous-graphes $f_{\Delta}$ à partir de $\Delta_{1}, \Delta_{2}$, et $f_{\Delta 1}$, $f_{\Delta 2}$ respectivement tels que $f$ est un isomorphisme de sous-graphes de $\left(G_{1} \cup G_{2}\right)$ à $G_{I}$. Soient $f_{1}=\left(\Delta_{1}, f_{\Delta 1}\right)$ et $f_{2}=\left(\Delta_{2}, f_{\Delta 2}\right)$. Alors :

$$
f_{\Delta}(v)= \begin{cases}f_{\Delta 1}(v) & \text { if } v \in V_{1} \\ f_{\Delta 2}(v) & \text { if } v \in V_{2}\end{cases}
$$




$$
\Delta=\Delta_{1}+\Delta_{2}+\Delta_{E}
$$

$\Delta_{E}$ est construite de la manière suivante : pour chaque paire $\left(v_{i}, w_{j}\right), v_{i} \in$ $V_{1}, w_{j} \in V_{2}$, s'il y a un $\operatorname{arc} e_{I}=\left(f_{\Delta 1}(v i), f_{\Delta 2}(w j)\right) \in G_{I}$, alors un $\operatorname{arc}(v i, w j)$ doit etre inséré dans $\Delta_{E}$.

Si $C_{1}$ et $C_{2}$ sont les coûts des isomorphismes avec tolérance d'erreur $f_{1}$ et $f_{2}$ respectivement, alors le coût de l'isomorphisme avec tolérance d'erreurs $f$ est : $C(f)=C_{1}+C_{2}+C\left(\Delta_{E}\right)$.

Pour résumer, si deux graphes cible $G_{1}$ et $G_{2}$ sont appariés à des sous-graphes disjoints du graphe d'entrée, il est possible de construire un graphe qui est leur composition pour trouver un meilleur appariement pour le graphe d'entrée.

\section{L'appariement des protocoles de conversation}

Dans cette section, nous illustrons l'utilisation de l'algorithme d'appariement de graphes avec tolérance d'erreurs par l'appariement des protocoles de conversation dérivés du standard de WSCL (Banerji et al., 2002). Nous donnons d'abord une vue d'ensemble du processus d'appariement et ensuite nous discutons chaque étape en détail. La même approche peut être appliquée pour d'autres modèles, tant que le protocole de conversation peut être transformé en graphe d'une manière unique (des représentations équivalentes d'un protocole de conversation sont réduites au même graphe). WSCL est un langage simple pour la définition des conversations, il offre les constructions de base pour modéliser l'ordonnancement des interactions ou des opérations décrites dans une interface de service. Ainsi il complète la définition de l'interface du service en indiquant l'ordre d'invocation des opérations. Une conversation dans WSCL est spécifiée en utilisant les constructions de base suivantes :

- Les interactions modélisent les actions de la conversation comme des échanges de documents entre deux participants. WSCL permet la modélisation de cinq types d'interactions : Send (le service envoie un document en sortie); Receive (le service reçoit un document d'entrée); SendReceive (le service envoie un document en sortie et ensuite il attend la reception d'un document en entrée comme réponse); ReceiveSend (le service reçoit un document en entrée et puis envoie un document en sortie); Empty (ne contient aucun document échangé, mais est utilisé seulement pour modéliser le début et la fin d'une conversation.)

- Les transitions indiquent les contraintes d'ordre entre les interactions.

Chaque interaction indique le type (schémas) de document XML qui est attendu en entrée ou est produit en sortie.

Le processus d'appariement de protocoles de conversation est composé des étapes suivantes. D'abord, les protocoles de conversation à comparer sont transformés en graphes. Après, les graphes sont étendus afin d'avoir le même niveau de granularité et l'algorithme d'appariement est appliqué. Finalement, les niveaux de granularité sont 
comparés et les coûts correspondant aux différences identifiées sont ajoutés à la distance totale.

L'architecture du système réalisant l'appariement basé sur le comportement est présentée dans la figure 2. Le système se compose d'un analyseur qui transforme un document WSCL en graphe et un module d'analyseur de similarité qui évalue la similarité entre les graphes. L'analyseur de similarité est composé des éléments suivants (figure 2) :

- Module de décomposition qui applique l'opération de décomposition afin d'avoir le même niveau du granularité dans les deux graphes.

- Appariement de graphes qui prend comme entrées les graphes produits par le module de décomposition et trouve l'isomorphisme de sous-graphes avec tolérance d'erreurs avec le coût minimal.

- Module des règles de comparaison qui regroupe les fonctions de coût pour les opérations d'édition de graphe.

- Comparaison linguistique qui implante différents algorithmes pour trouver la similarité entre deux chaînes de caractères.

- Comparaison de la granularité qui vérifie si des opérations de composition/jointure sont nécessaires et ajoute leurs coût à la distance totale.

Dans les sections suivantes nous présentons en détail les fonctionnalités de chaque module.

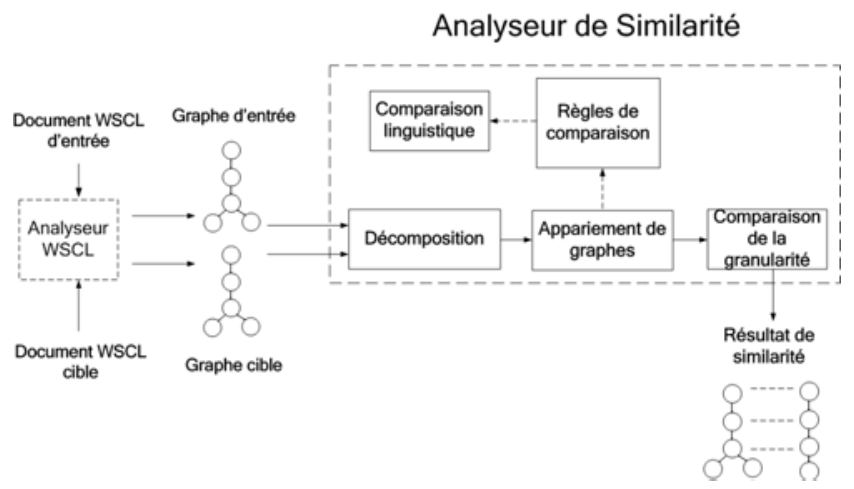

Figure 2. Architecture

\subsection{Transformation d'un protocole de conversation dans un graphe}

L'analyseur transforme un modèle de conversation WSCL en un graphe dont les sommets représentent des interactions et dont les arcs représentent des transitions.

Chaque noeud a les attributs suivants : nom, type d'interaction et documents. La figure 3 montre la correspondance entre les éléments d'un protocole WSCL et les éléments du graphe résultant. 


\begin{tabular}{|c|c|}
\hline WSCL & Graphe \\
\hline Interaction & Sommet \\
\hline Type & attribut du sommet \\
\hline Document d'entrée & attribut du sommet \\
\hline Document de sortie & attribut du sommet \\
\hline Id & étiquette \\
\hline Transition & Arc \\
\hline Transition condition & attribut de l'arc \\
\hline
\end{tabular}

Figure 3. Correspondances entre les éléments WSCL et un graphe

\subsection{Décomposition des interactions}

La deuxième étape dans l'appariement est l'extension des graphes. Les opérations de décomposition sont appliquées afin d'avoir le même niveau de granularité dans les deux graphes. L'opération de décomposition dépend du méta-modèle des protocoles à comparer. Par exemple, pour le méta-modèle de WSCL, il est possible que dans un protocole une interaction soit modélisée par une interaction SendReceive tandis que dans le deuxième protocole la même fonctionnalité est réalisée en utilisant une interaction Send suivie par Receive. Ainsi, le module de décomposition transformera des interactions de type ReceiveSend dans des interactions atomiques : Send et Receive. Une interaction SendReceive est décomposée en une interaction Send suivie par une interaction Receive de la façon suivante :

- tous les arcs ayant comme destination l'interaction SendReceive auront comme destination l'interaction Send;

- tous les arcs ayant comme source l'interaction SendReceive auront comme source l'interaction Receive;

- un arc sera ajouté entre l'interaction Send et l'interaction Receive;

- si l'interaction SendReceive a un document de sortie $a$ et un document d'entrée $b$, alors l'interaction Send aura $a$ comme document de sortie et l'interaction Receive aura $b$ comme document d'entrée.

D'une manière similaire, une interaction ReceiveSend est décomposée en une interaction Receive suivie par une interaction Send.

Cette fonction de décomposition est spécifique au méta-modèle WSCL. Pour d'autres applications, l'utilisateur peut indiquer une fonction différente de décomposition. La fonction de décomposition a toujours la même signature : elle prend comme argument un sommet et renvoie deux sommets résultant de la décomposition (qui sont supposés être séquentiels). Le comportement de la fonction est spécifique à l'application (méta-modèle des protocoles à comparer) et spécifie comment les étiquettes et les attributs des deux sommets sont obtenus à partir du sommet décomposé. 


\subsection{Appariement des Graphes}

Ce module implémente l'algorithme pour la détection de l'isomorphisme de sousgraphe avec tolérance d'erreur (Messmer, 1995). La détection de l'isomorphisme de sous-graphe est basée sur un algorithme de recherche dans un espace d'états similaire à A* (Shapiro et al., 1981). L'idée fondamentale d'une recherche dans un espace d'états est d'avoir des états représentant des solutions partielles du problème donné et de définir des transitions d'un état à l'autre telles que le dernier état représente une solution plus complète que l'état précédent. Pour chaque état $s$ il y a une fonction $f$ d'évaluation qui décrit la qualité de la solution représentée. Les états sont explorés selon la valeur de $f$. Dans le cas de la détection d'isomorphismes de sous-graphe, étant donnés un graphe cible $G$ et un graphe d'entrée $G_{I}$, un état $s$ dans l'espace de recherche représente un appariement partiel de $G$ à $G_{I}$. Chaque appariement partiel implique un certain nombre d'opérations d'édition dont le coût peut être utilisé pour définir la fonction $f$ d'évaluation. En d'autres termes, l'algorithme commence par comparer le premier noeud de $G$ avec tous les noeuds de $G_{I}$ et choisit le meilleur mapping (avec le coût minimal) (voir l'algorithme 1, Ligne 1). Ceci représente un mapping partiel qui sera élargi en ajoutant un noeud à la fois (ligne 7 ). La fonction qui compare deux noeuds (VertexMatch) sera expliquée dans la section suivante.

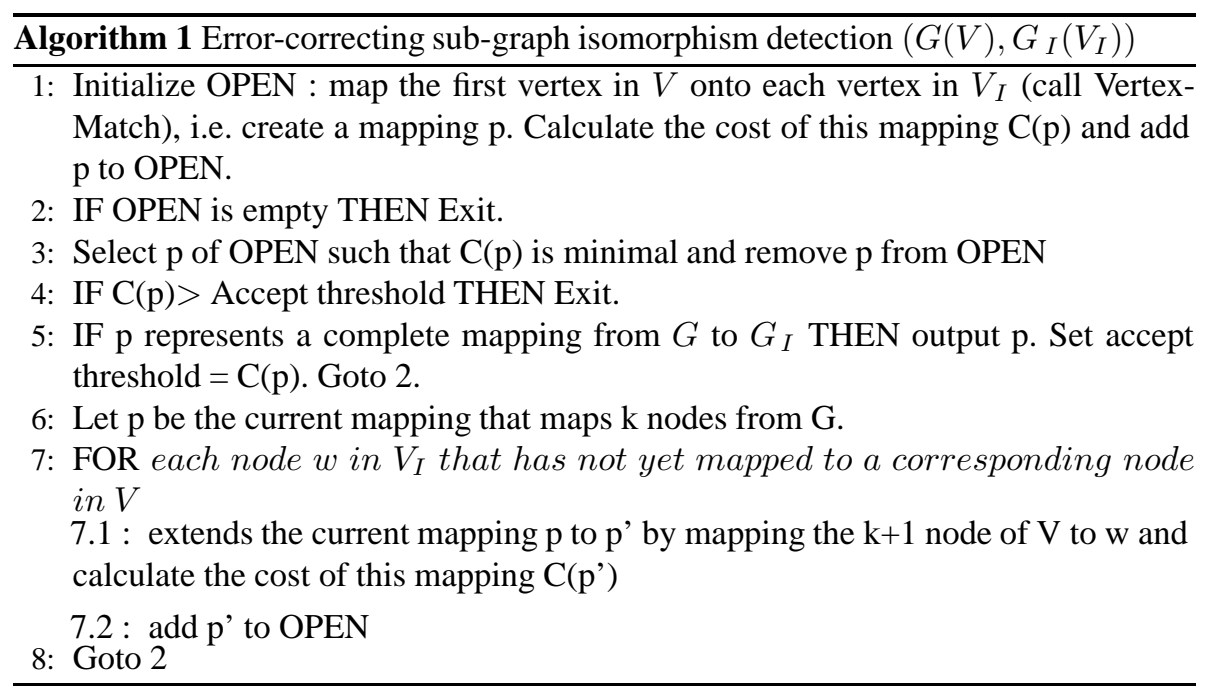

Le coût du mapping C(p') (ligne 7.1) exprime le coût d'éteindre le mapping actuel $p$ en traitant le sommet suivant du graphe requête. Elargir le mapping actuel en ajoutant le mapping entre le noeud $v$ du graphe requête et le noeud $w$ du graphe cible (qui n'a pas été encore mise en correspondance) implique des opération d'édition de noeud et d'arc. Premièrement, l'étiquette et les attributs du sommet $v$ doivent être substitués par l'étiquette et les attributs du $w$. Le coût de ces opérations est calculé par la fonction VertexMatch expliquée dans la section suivante. Deuxièmement, pour chaque mapping $\left(v^{\prime}, w^{\prime}\right) \in p$ on doit garantir que chaque arc $\left(v^{\prime}, v\right)$ dans le graphe cible peut 
être mis en correspondance avec un $\operatorname{arc}\left(w^{\prime}, w\right)$ dans le graphe d'entrée grâce à des opérations d'édition.

Le processus se termine soit quand un état représentant un isomorphisme de sousgraphe avec tolérance d'erreurs de $G$ à $G_{I}$ a été atteint, soit quand tous les états de l'espace de recherche ont des coûts d'édition qui excèdent un certain seuil donné (ligne $4)$.

\subsection{Règles de Comparaison}

Le module des Règles de comparaison contient toutes les fonctions dépendantes de l'application permettant de calculer le coût des opérations d'édition de graphes. Ces fonctions sont utilisées par le module d'appariement pour calculer la distance entre les graphes. Pour permettre des applications ayant des fonctions de coût spécialisées, des fonctions de coût définies par l'utilisateur peuvent être enregistrées dans ce module. Dans ce qui suit, nous expliquons la fonction de coût utilisée pour l'appariement de protocoles de conversation.

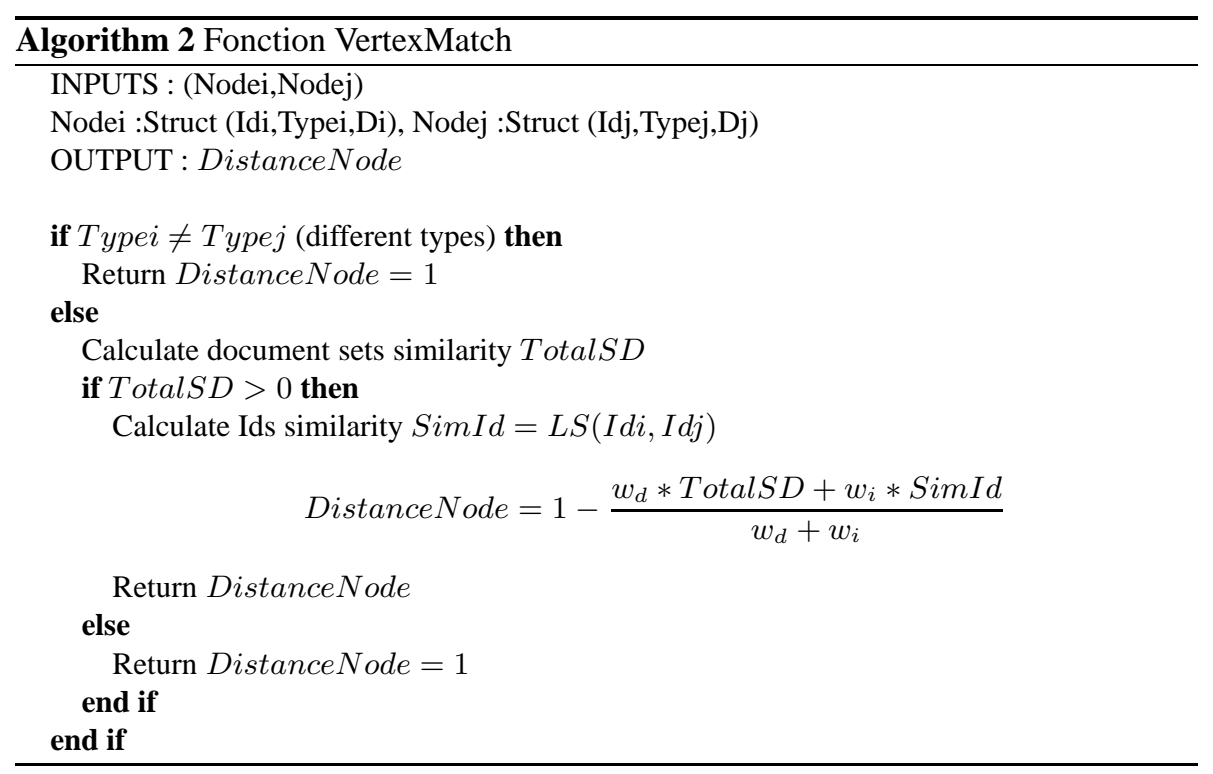

Les coûts pour l'insertion, la suppression des arcs et des sommets peuvent être fixés à des constantes. Le coût pour l'édition d'un sommet est calculé par la fonction VertexMatch (voir l'algorithme 2). Comme les sommets représentent des interactions WSCL, ce coût exprime la distance entre deux interactions WSCL. Chaque interaction a une étiquette $(I d)$ et deux attributs : le type d'interaction (Type) et l'ensemble de documents $(D)$ (d'entrée ou de sortie). Si deux interactions ont le même type, la similarité de l'ensemble de documents Total $S D$ est calculée; si cette valeur n'est 
pas nulle (TotalSD>0), la fonction calcule la similarité des noms d'interactions (SimId).

La fonction $S D(D i, D j)$ où $D i, D j$ sont l'ensemble des documents des $N o d e i$ et Nodej respectivement, calcule le meilleur mapping qui peut être obtenu entre les documents des deux ensembles.

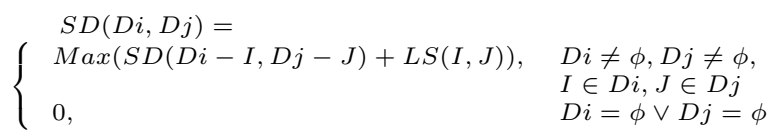

Le nombre de mappings trouvés est $\operatorname{Min}(|D i|,|D j|)$. La fonction $L S$ calcule la similarité linguistique entre les noms des documents, elle est expliquée dans la section suivante.

Finalement, la similarité totale de l'ensemble des documents est :

$$
\operatorname{Total} S D=\frac{S D(D i, D j)}{k}
$$

où, $\mathrm{k}=$ Nombre de documents de l'ensemble $D i$.

Les poids $w_{d}$ and $w_{i}$ indiquent la contribution de Total $S D$ (similarité des documents échangés) et $\operatorname{SimId}$ (similarité des noms des interactions) respectivement dans la distance totale DistanceNoeud $(0 \leq w d \leq 1$ and $0 \leq w i \leq 1)$.

\subsection{Comparaison Linguistique}

Le module Comparaison Linguistique calcule la similarité linguistique entre les noms des deux étiquettes (Patil et al., 2004).

Les étiquettes sont souvent constituées par un mot ou par une combinaison des mots et peuvent contenir des abréviations. Pour obtenir une distance linguistique entre deux chaînes de caractères, nous utilisons des algorithmes existants : NGram, Check synonym, and Check abbreviation. L'algorithme NGram estime la similarité en fonction du nombre commun de qgrams entre les deux noms d'étiquettes (Angell et al., 1983). L'algorithme Check synonym utilise un dictionnaire linguistique (par exemple Wordnet (Miller, 1995) dans notre implémentation) pour trouver les synonymes entre les noms d'étiquettes et l'algorithm Check abbreviation utilise un dictionnaire d'abréviations selon le domaine d'application. Si chaque algorithme renvoie 1 , il y a un appariement exact. Si tous les algorithmes renvoient 0 , alors il n'y a aucun appariement entre les étiquettes. Si la valeur de NGram et la valeur de Check abbreviation sont égales à 0 , et Check Synonym est entre 0 et 1 , la valeur finale de la similarité linguistique sera égale à la valeur Check Synonym. Finallement, si les trois 
valeurs d'algorithmes sont entre 0 et 1 , la similarité LS ((Patil et al., 2004)) est égale à la moyenne des trois valeurs :

$$
L S= \begin{cases}1 & \text { if }(m 1=1 \vee m 2=1 \vee m 3=1) \\ m 2 & \text { if }(0<m 2<1 \wedge m 1=m 3=0) \\ 0 & \text { if }(m 1=m 2=m 3=0) \\ \frac{m 1+m 2+m 3}{3} & \text { if } m 1, m 2, m 3 \in(0,1)\end{cases}
$$

où $\mathrm{m} 1=\operatorname{Sim}(\mathrm{NGram}), \mathrm{m} 2=\operatorname{Sim}($ Synonim Matching $)$ and $\mathrm{m} 3=\operatorname{Sim}($ Abbreviation Expansion).

\subsection{Comparaison des niveaux de granularité}

L'algortihm d'appariement de graphes avec tolérance d'erreur est appliqué aux graphes qui ont été préalablement étendus, c'est-à-dire ils contiennent seulement des interactions de type Send et Receive. Le module de comparaison de la granularité vérifie si les interactions qui ont été mises en correspondance par l'algorithme d'appariement ont le même niveau de granularité dans les deux modèles. Par exemple, supposons que le graphe requête contient une interaction SendReceive. Ceci a été décomposé par le module de décomposition dans une interaction Send suivie par une interaction Receive qui ont été mises en correspondance avec deux interactions de même type dans le graphe cible. Si ces interactions étaient atomiques dans le graphe cible, le coût de l'opération de jointure doit être ajouté à la distance totale des graphes (ligne 5 dans le tableau de la Figure 4).

\begin{tabular}{|c|c|c|}
\hline $\begin{array}{l}\text { Type d'interaction } \\
\text { graphe d'entrée }\end{array}$ & $\begin{array}{l}\text { Type d'interaction } \\
\text { graphe cible }\end{array}$ & $\begin{array}{c}\text { Coût pour la } \\
\text { diff. de granularité }\end{array}$ \\
\hline $\mathrm{S}$ atomic & $\mathrm{S}$ atomic & 0 \\
\hline $\mathrm{R}$ atomic & $\mathrm{R}$ atomic & 0 \\
\hline SR & $\overline{S R}$ & 0 \\
\hline $\mathrm{RS}$ & $\mathrm{RS}$ & 0 \\
\hline SR (or RS) & $\mathrm{S}$ atomic $+\mathrm{R}$ atomic & $\bar{c}$ \\
\hline SR (or RS) & $\mathrm{S}$ nonat $+\mathrm{R}$ nonat & $c_{d}+c_{j}$ \\
\hline SR (or RS) & $\begin{array}{l}\mathrm{S} \text { nonat }+\mathrm{R} \text { atomic or } \\
\mathrm{S} \text { atomic }+\mathrm{R} \text { nonat }\end{array}$ & $c_{d} / 2+c_{j}$ \\
\hline $\mathrm{S}$ atomic & S nonatomic & $c_{d} / 2$ \\
\hline $\mathrm{R}$ atomic & $\mathrm{R}$ nonatomic & $c_{d} / 2$ \\
\hline
\end{tabular}

$\mathrm{S}=$ Send, R=Receive, $\mathrm{SR}=$ SendReceive, $\mathrm{RS}=$ ReceiveSend

Figure 4. Coûts pour les différences de granularité

Les coûts qui doivent être pris en compte dans la distance totale de graphe pour les différents types de différences de granularité (interactions atomiques versus non atomiques dans le graphe cible et requête) sont présentés dans la Figure 4. (Le tableau ne présente pas les cas où une des interactions d'une interaction décomposée a été supprimée par une opération d'édition.) Si les interactions mises en correspondance ont le même niveau de granularité (elles sont toutes les deux atomiques ou non-atomiques) il n'y a pas de coût supplémentaire à ajouter à la distance d'édition de sous-graphe. 
Un cas plus compliqué (la ligne 7 dans le tableau de la Figure 4) est quand une interaction SendReceive $S R_{I}$ dans le graphe d'entrée est mise en correspondance avec une interaction atomique Send $S_{M}$ suivie par une interaction Receive $R_{M}$ qui n'est pas atomique (appartient à une interaction SendReceive $S R_{M}$ ) dans le graphe cible. Dans ce cas, le coût est $c_{j}+c_{d} / 2\left(c_{j}=\right.$ coût pour la jointure de $S_{M}$ et $R_{M} ; c_{d} / 2=$ coût pour obtenir $R_{M}$ en décomposant l'interaction $S R_{M}$ dans le graphe cible).

\subsection{Exemple}

Supposons qu'on veuille trouver la similarité entre deux services de réservation de hôtel dont les protocoles de conversations ont été décrits en utilisant WSCL. Le protocole de conversation du premier service est composé des interactions suivantes : ReservationRequest (interaction de type : Receive), RequestCatalog (type : Receive) suivies par SendCatalog (Type : Send) et CheckAvailability (Type : ReceiveSend). Le protocole de conversation du deuxième service a la séquence d'interactions suivante : ReservationRQ (Type : Receive), Availability (Type : ReceiveSend), Cata$\log$ (Type : ReceiveSend). Le système transforme chaque document WCSL dans un graphe (graphe d'entrée et graphe requête dans la Figure 5). Ensuite les graphes sont étendus (Graphe d'entrée décomposé et graphe cible décomposé dans la Figure 5) en utilisant la fonction de décomposition.

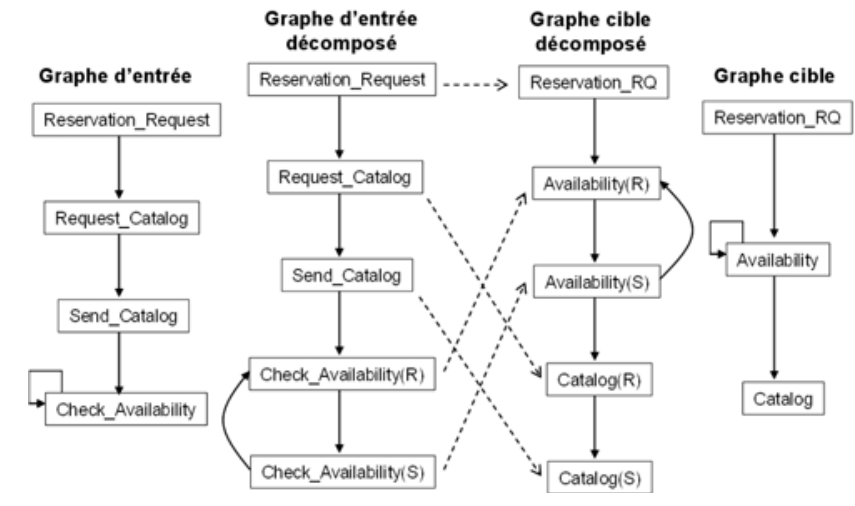

Figure 5. Exemple

Les lignes pointillées dans la figure Figure 5 représentent les mappings trouvés par le système entre les deux graphes en utilisant les règles de comparaison. Finalement, le coût correspondant aux différences de granularité est ajouté à la distance totale de graphes. (L'interaction Catalog dans le graphe cible doit être décomposée pour être apparié avec les deux interactions correspondantes dans le graphe d'entrée).

En conclusion, le script d'édition va montrer que les deux graphes sont similaires, mais ils ont les différences structurelles suivantes : les interactions RequestCatalog (Type : Receive) et SendCatalog (Type : Send) sont modélisées comme une seule interaction SendReceive dans le graphe cible; les interactions pour vérifier la disponibilité 
et pour demander le catalogue ne sont pas exécutées dans le même ordre dans les deux protocoles.

\section{Implémentation et expérimentations}

Dans cette section, nous présentons le prototype que nous avons développé pour l'appariement de protocoles de conversation et les résultats de quelques expérimentations que nous avons effectuées.

Nous avons implémenté un système ayant l'architecture présentée dans la section précédente. Le système est disponible aussi comme un service qui prend comme entrées deux documents WSCL et calcule leur similarité. Il renvoie aussi la séquence d'opérations d'édition nécessaire pour transformer le protocole d'entrée pour le rendre similaire au protocole cible.

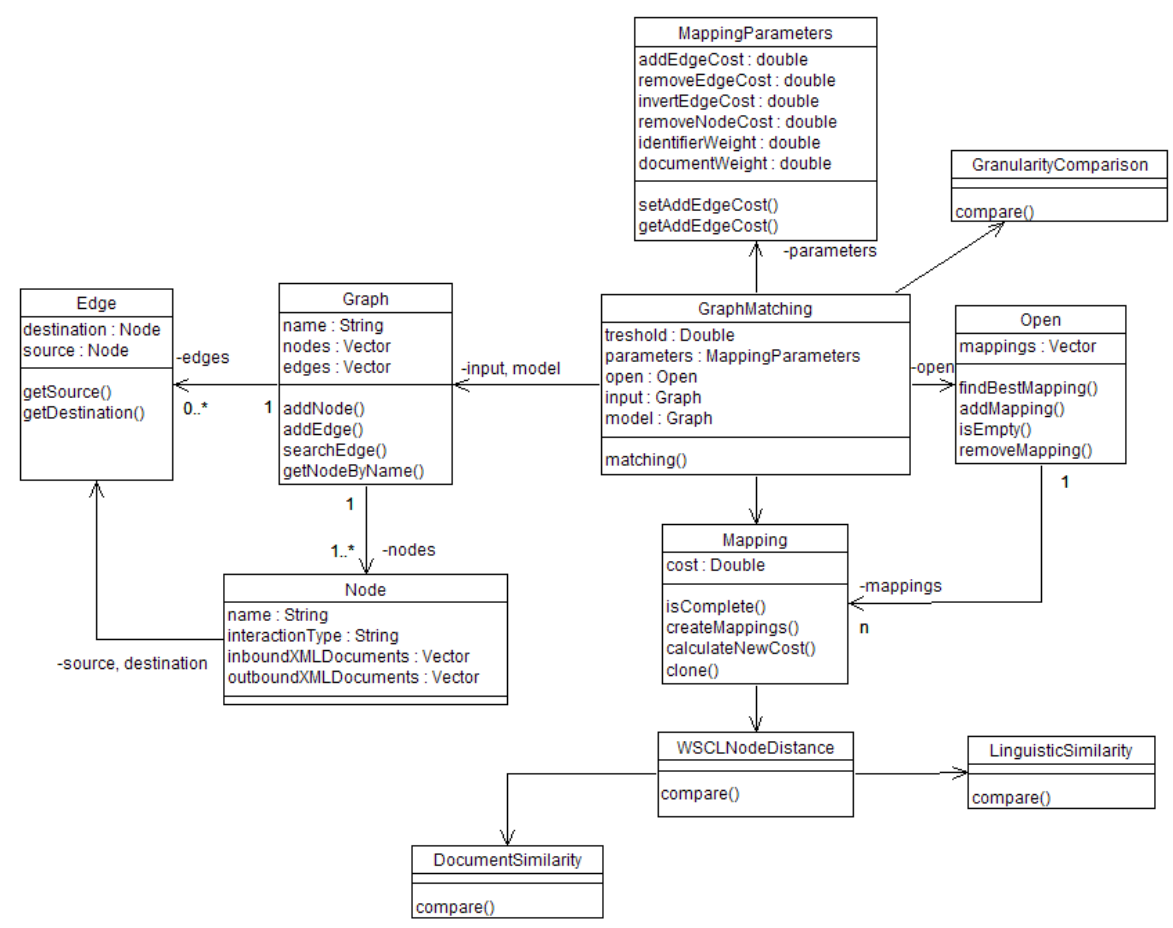

Figure 6. Le diagramme de classes du prototype

La Figure 6 montre le diagramme de classe du prototype. Les classes Graph, Edge et Node forme une représentation objet d'un graphe dirigé et étiqueté. La classe Node inclue les attributs qui modélisent une interaction WSCL (nom, type, documents d'entrée et de sortie). La classe Mapping représente un mapping partiel entre les deux 
graphes. La classe Open stocke l'ensemble de mappings. A chaque étape de l'algorithme d'appariement de graphes, le mapping de la liste Open ayant le coût minimale est sélectionné et il est étendu. L'algorithme s'arrête quand la liste de mappings est vide ou quand le coût de chaque mapping dépasse un seuil fixé.

La classe MatchingParameters contient les paramètres pour les opérations d'édition qui peuvent être affectés par l'utilisateur. La comparaison entre deux interactions WSCL (WSCLNodeDistance) est réalisée en utilisant deux classes : une classe trouve la similarité entre les deux ensembles de documents et la deuxième classe calcule la similarité linguistique des noms. L'algorithme d'appariement est implémenté dans la classe GraphMatching. L'évaluation du coût correspondant aux différences de granularité est réalisée par la classe GranularityComparison.

Le prototype a été implémenté en utilisant le langage de programmation Java et l'environnement de développement intégré (IDE) Eclipse. Il utilise des API Xerces pour les documents WSCL dans le module du parseur. Pour la représentation visuelle des graphes les API JGraph et JgraphLayout ont été utilisés. Ces API fournissent des fonctions utiles pour dessiner des graphes comme des composants compatibles Swing. Le module de comparaison linguistique accède le dictionnaire Wordnet en utilisant les API Java Word Net Library (JWNL).

La figure 7 montre l'interface graphique dans laquelle l'utilisateur peut choisir les documents WSCL d'entrée et peut visualiser les graphes correspondants. L'interface permet également à l'utilisateur d'affecter des coûts spécifiques pour les opérations d'édition de graphe (interactions et transitions), le coût pour les différences de granularité, les poids pour la similarité des noms des interactions et des documents ( $W i$ et $W d$ ) dans la similarité totale, et le seuil pour la distance d'édition de graphe (Acceptable value). L'interface montre également la décomposition des graphes d'entrée et cible. Les résultats de l'algorithme d'appariement sont affichés dans une autre fenêtre sous une forme graphique et textuelle.

La complexité théorique de l'algorithme d'appariement de graphes (Messmer, $1995)$ est $\left(O\left(m^{2} n^{2}\right)\right.$ dans le cas le plus favorable (quand la distance entre le graphe d'entrée et le graphe cible est minimale) et $O\left(m^{n} n\right)$ dans le cas le plus défavorable ( $\mathrm{m}=$ le nombre de noeuds du graphe d'entrée; $\mathrm{n}=$ le nombre de noeuds du graphe cible). Malgré le coût exponentiel de l'algorithme, nos expérimentations ont montré qu'il peut être utilisé pour des graphes ayant moins de 50 noeuds, ce qui correspond à un problème de taille raisonnable (Figure 8).

Un problème important pour l'algorithme d'appariement de graphes est la paramétrisation de la fonction de coût. La qualité de l'appariement obtenu et le comportement de l'algorithme dépendent fortement de cette fonction. Selon (Bunke et al., 2000), le choix des coûts des opérations d'édition dépend de l'application et se fait de manière ad hoc, guidée par des heuristiques et l'intuition. Nos expériences ont montré que des résultats de bonne qualité peuvent être obtenus avec la paramétrisation suivante de 


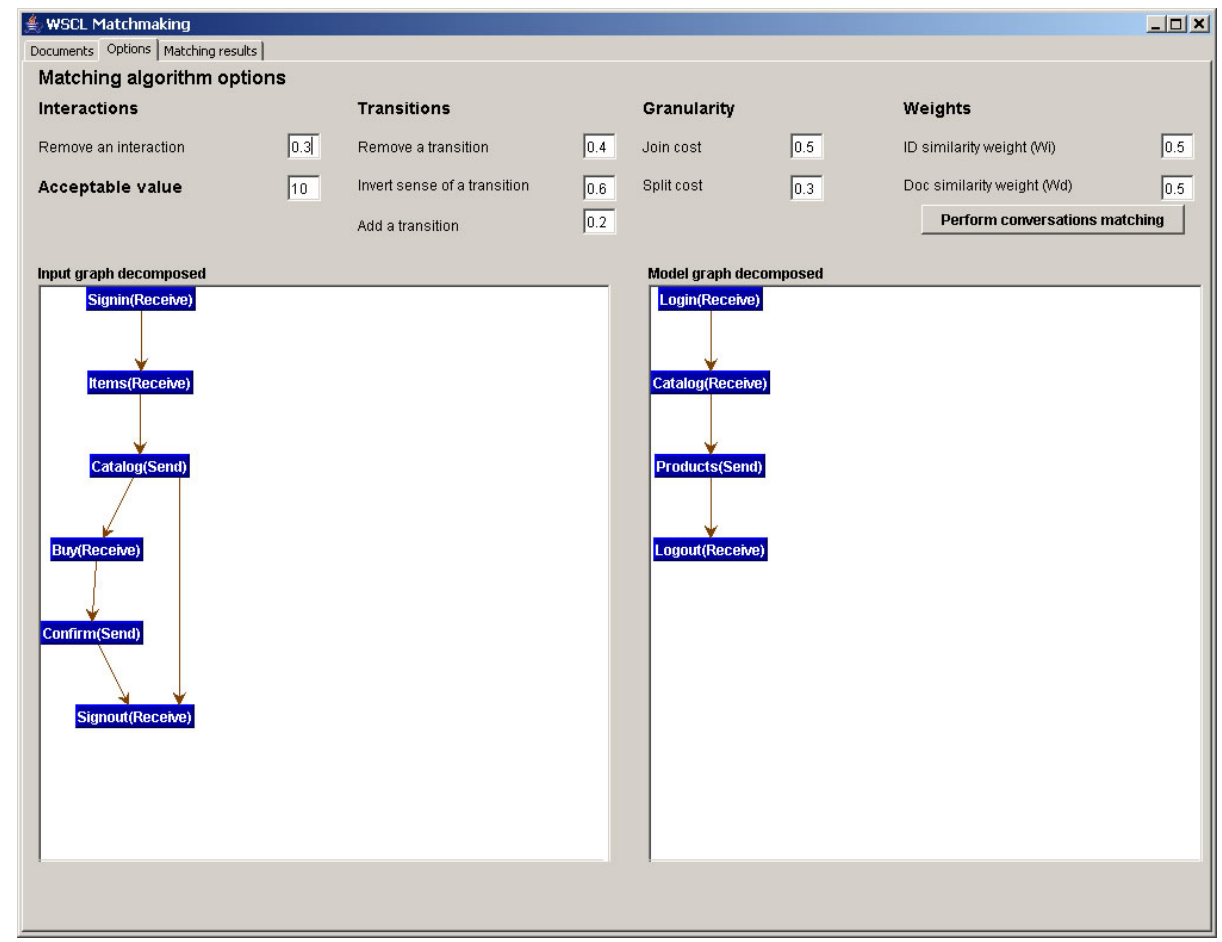

Figure 7. Interface Paramétrisation

la fonction de coût : coût de suppression d'un noeud $=0.8$, coût de suppression d'un $\operatorname{arc}=0.2$, coût d'insertion d'un arc $=0.2$.

\section{Conclusion}

Dans cet article nous avons proposé une solution pour la découverte de services, basée sur des spécifications comportementales. D'abord nous avons motivé la nécessité de retrouver des services en se basant sur leur modèle de conversation. En utilisant un formalisme de représentation à base de graphes pour des services, nous avons proposé d'utiliser un algorithme d'appariement de graphes avec tolérance d'erreurs afin de permettre un appariement partiel. En partant de la distance classique d'édition de graphes, nous avons proposé deux nouvelles opérations pour prendre en compte les différences des niveaux de granularité qui peuvent apparaître dans deux modèles. Nous avons défini une mesure de similarité pour l'appariement de services basé sur le comportement et nous avons montré comment composer deux graphes cibles afin de répondre aux besoins de l'utilisateur. Nous avons illustré notre approche pour l'appariement des service basé sur le comportement en utilisant des protocoles de conversation WSCL. 


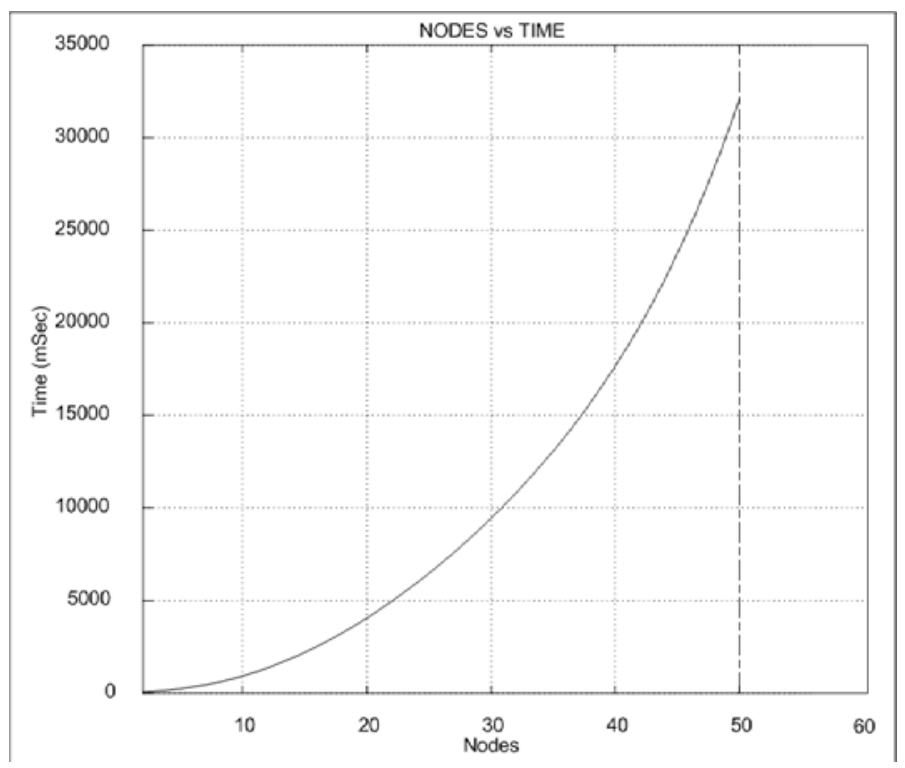

Figure 8. Temps d'exécution

Nos travaux en cours portent sur la généralisation de l'opération d'édition de graphes pour la composition et la jointure pour traiter la situation où un noeud du premier graphe correspond à un sous-graphe du deuxième graphe. Cette situation apparaît quand le premier service a une seule opération (activité) pour implémenter une certaine fonctionnalité, pendant que dans le deuxième service la même fonctionnalité est réalisée en utilisant plusieurs opérations.

La prochaine étape de ce travail consiste à proposer une méthode permettant de trouver un service dans une librairie de services. Pour comparer un protocole de conversation avec un ensemble de protocoles dans une librairie, des problèmes de performance liés au temps d'exécution doivent être résolus. Nous évaluerons aussi de manière expérimentale la qualité de la méthode de découverte basée sur le comportement.

\section{Bibliographie}

Angell R. C., Freund G. E., Willett P., « Automatic Spelling Correction Using a Trigram Similarity Measure », Information Processing and management, vol. 19, $\mathrm{n}^{\circ}$ 4, p. 255-261, 1983.

Banerji A., Bartolini C., Beringer D., Chopella V., Govindarajan K., Karp A., Kuno H., Lemon M., Pogossiants G., Sharma S., Williams S., « Web Services Conversation Language (WSCL) $1.0 », W 3 C, 2002$. 
Benatallah B., Casati F., Grigori D., Motahari Nezhad H. R., Toumani F., « Developing Adapters for Web Services Integration », Proc. of Conference on Advanced Information Systems Engineering (CAISE), 2005.

Benatallah B., Casati F., Toumani F., « Analysis and Management of Web Services Protocols », Proc. of International Conference on Conceptual Modeling (ER), 2004a.

Benatallah B., Casati F., Toumani F., «Web services conversation modeling : A Cornerstone for E-Business Automation », IEEE Internet Computing, 2004b.

Benatallah B., Hacid M., Rey C., Toumani F., « Semantic Reasoning for Web Services Discovery », Proc. of WWW Workshop on E-Services and the Semantic Web, 2003.

Bernstein A., Klein M., « Towards High-Precision Service Retrieval», Proc. of Int. Semantic Web Conference (ISWC), 2002.

Bordeaux L., et al., «When are two Web Services Compatible ?», Proc. of TES, 2004.

Bunke H., « Recent developments in graph matching », Proc. of 15th Int. Conf. on Pattern Recognition, p. 117 - 124, 2000.

Bunke H., Jiang X., « Graph matching and similarity », Intelligent Systems and Interfaces, 2000.

Cardoso J., Sheth A., « Semantic E-Workflow Composition », Journal of Intelligent Information Systems, vol. 21, p. 191-225, 2003.

Dong L., Halevy A., Madhavan J., Nemes E., , Zhang J., « Similarity Search for Web Services », Proc. of International Conference on Very Large Data Bases (VLDB), 2004.

Foster I., Voeckler J., Wilde M., Zhao Y., « Chimera : A Virtual Data System for Representing, Querying and Automating Data Derivation », Proc. of 14th Conf. on Scientific and Statistical Database Management, 2002.

Foster I., Voeckler J., Wilde M., Zhao Y., « The Virtual Data Grid : A New Model and Architecture for Data-Intensive Collaboration », Proc. of CIDR - Conf. on Innovative Data System Research, 2003.

Grigori D., Bouzeghoub M., « Service retrieval based on behavioral specification », IEEE International Conference on Services Computing (SCC), n.d.

Kawamura T., De Blasio J., Hasegawa T., Paolucci M., Sycara K., « A Preliminary Report of a Public Experiment of a Semantic Service Matchmaker combined with a UDDI Business Registry », Proc. of 1st International Conference on Service Oriented Computing (ICSOC), 2003.

Messmer B., Graph Matching Algorithms and Applications, PhD thesis, University of Bern, 1995.

Miller G., « WordNet : A Lexical Database for English », Communications of the ACM, vol. 38, n 11 , p. 39-41, 1995.

Paolucci M., Kawamura T., Payne T. R., Sycara K., « Semantic Matching of Web Services Capabilities », Proc. of First International Semantic Web Conference (ISWC), 2002.

Patil A., Oundhakar S., Sheth A., Verna K., « METEOR-S Web Service Annotation Framework », Proc. of WWW Conference, 2004.

Piccinelli G., Di Vitantonio G., Mokrushin L., « Dynamic service aggregation in electronic marketplaces », Computer Networks, 2001.

Ponnekanti S. R., Fox A., « Interoperability among Independently Evolving Web Services », Proc. of ACM/IFIP/USENIX International Middleware Conference, 2004. 
S. Bansal S., Vidal J. M., « Matchmaking of web services based on the DAML-S service model », Proc. of Int. Joint Conference on Autonomous Agents and Multiagent Systems, p. 926927, 2003

Shapiro L. G., Haralick R. M., « Structural descriptions and inexact matching », IEEE Trans. Pattern Anal. Mach. Intell., 1981.

Shen Z., Su J., « Web Services Discovery Based on Behavior Signatures », Proc. of IEEE International Conference on Services Computing, 2005.

Trastour D., Bartolini C., Gonzalez-Castillo J., « A Semantic Web Approach to Service Description for Matchmaking of Services », Proc. of Int. Semantic Web Working Symposium (SWWS), 2001.

Wombacher A., Mahleko B., Fankhauser P., « A Grammar-Based Index for Matching Business Processes », Proc. of IEEE International Conference on Web Services, p. 21-30, 2005.

Wombacher A., Mahleko B., Fankhauser P., Neuhold E., « Matchmaking for Business Processes based on Choreographies », Proc. of IEEE International Conference on e-Technology, eCommerce and e-Service, 2004.

Wu J., Wu Z., « Similarity-based Web Service Matching », Proc. of IEEE International Conference on Services Computing, 2005.

Zdravkovic J., P. Johanesson P., « Cooperation of Processes through Message Level Agreement », Proc. of Int. Conf. On Advanced Information Systems Engineering (CAISE), 2004. 


\section{ANNEXE POUR LE SERVICE FABRICATION \\ A FOURNIR PAR LES AUTEURS AVEC UN EXEMPLAIRE PAPIER \\ DE LEUR ARTICLE ET LE COPYRIGHT SIGNE PAR COURRIER \\ LE FICHIER PDF CORRESPONDANT SERA ENVOYE PAR E-MAIL}

1. ARTICLE POUR LA REVUE :

ISI. Volume $8-n^{\circ} 2 / 2005$

2. Auteurs :

Juan Carlos Corrales — Daniela Grigori - Mokrane Bouzeghoub

3. TITRE DE L'ARTICLE :

Découverte de services basée sur leurs protocoles de conversation

4. Titre ABRÉGÉ POUR LE HAUT DE PAGE MOINS DE 40 SIGNES :

Découverte de services

5. DATE DE CETTE VERSiON :

15 mars 2007

6. CoOrdonnÉEs des Auteurs :

- adresse postale :

Laboratoire PRiSM, Université de Versailles Saint-Quentin en Yvelines

45 avenue des Etats-Unis, 78035 Versailles Cedex, FRANCE

\{Juan-Carlos.Corrales-Munoz,Daniela.Grigori,Mokrane.Bouzeghoub\} @ prism.uvsq.fr

- téléphone : 0139254053

- télécopie : 0139254057

- e-mail : guillaume.laurent@ens2m.fr

7. LOGICIEL UTILISÉ POUR LA PRÉPARATION DE CET ARTICLE :

LTEX, avec le fichier de style article-hermes.cls, version $1.23 \mathrm{du} 17 / 11 / 2005$.

8. Formulaire DE COPYRIGHT:

Retourner le formulaire de copyright signé par les auteurs, téléchargé sur :

http://www.revuesonline.com

SERVICE ÉDITORIAL - HERMES-LAVOISIER

14 rue de Provigny, F-94236 Cachan cedex

Tél. : 01-47-40-67-67

E-mail : revues@lavoisier.fr

Serveur web : http://www.revuesonline.com 